\title{
Rectal Swabs as an Alternative Sample Collection Method to Bulk Stool for the Real-Time PCR Detection of Giardia duodenalis
}

\author{
Jacqueline R. M. A. Maasch, ${ }^{1}$ Ahmed M. Arzika, ${ }^{2}$ Catherine Cook, ${ }^{3}$ Elodie Lebas, ${ }^{3}$ Nils Pilotte, ${ }^{1,4}$ Jessica R. Grant, ${ }^{1}$ \\ Steven A. Williams, ${ }^{1,4}$ Jeremy D. Keenan, ${ }^{3}$ Thomas M. Lietman, ${ }^{3}$ and Kristen Aiemjoy ${ }^{3,5 *}$ \\ ${ }^{1}$ Department of Biological Sciences, Smith College, Northampton, Massachusetts; ${ }^{2}$ The Carter Center, Niamey, République du Niger; ${ }^{3}$ Proctor \\ Foundation, University of California San Francisco, San Francisco, California; ${ }^{4}$ Molecular and Cellular Biology Program, University of \\ Massachusetts, Amherst, Massachusetts; ${ }^{5}$ Division of Infectious Diseases and Geographic Medicine, Department of Medicine, Stanford University \\ School of Medicine, Stanford, California
}

\begin{abstract}
Though bulk stool remains the gold standard specimen type for enteropathogen diagnosis, rectal swabs may offer comparable sensitivity with greater ease of collection for select pathogens. This study sought to evaluate the validity and reproducibility of rectal swabs as a sample collection method for the molecular diagnosis of Giardia duodenalis. Paired rectal swab and bulk stool samples were collected from 86 children ages $0-4$ years living in southwest Niger, with duplicate samples collected among a subset of 50 children. Infection was detected using a previously validated real-time PCR diagnostic targeting the small subunit ribosomal RNA gene. Giardia duodenalis was detected in $65.5 \%$ (55/84) of bulk stool samples and $44.0 \%$ (37/84) of swab samples. The kappa evaluating test agreement was 0.81 (95\% Cl: 0.54-1.00) among duplicate stool samples $(N=49)$ and $0.75(95 \% \mathrm{Cl}: 0.47-1.00)$ among duplicate rectal swabs $(N=48)$. Diagnostic sensitivity was $93 \%$ (95\% Cl: $84-98)$ by bulk stool and $63 \%(95 \% \mathrm{Cl}: 49-75)$ by rectal swabs. When restricting to the lowest three quartiles of bulk stool quantitation cycle values (an indication of relatively high parasite load), sensitivity by rectal swabs increased to $78.0 \%$ (95\% Cl: $64-89, P<0.0001)$. These findings suggest that rectal swabs provide less sensitive and reproducible results than bulk stool for the real-time PCR diagnosis of G. duodenalis. However, their fair sensitivity for higher parasite loads suggests that swabs may be a useful tool for detecting higher burden infections when stool collection is excessively expensive or logistically challenging.
\end{abstract}

\section{INTRODUCTION}

The protozoan flagellate Giardia duodenalis (syn. Giardia intestinalis and Giardia lamblia) parasitizes the human small intestine and is the etiological agent of giardiasis, a globally prevalent diarrheal disease. The life cycle of $G$. duodenalis entails a dormant yet infectious cyst phase and a motile, replicating trophozoite phase, with its morphology typified by an adhesive disc significantly implicated in virulence. ${ }^{1-3} \mathrm{Ge}-$ nomic analyses suggest that $G$. duodenalis is a species complex comprised of eight distinct groups, assemblages $\mathrm{A}-\mathrm{H}$, with assemblages $\mathrm{A}$ and $\mathrm{B}$ commonly associated with human hosts. ${ }^{4,5}$ Although many infections are asymptomatic, giardiasis manifests with diverse symptomology and may contribute to physical and cognitive stunting in chronic cases. ${ }^{6}$ Children are often at high risk for G. duodenalis infection relative to other age-groups. ${ }^{7-11}$

Although microscopic assessment of ova, cysts, and parasites from bulk stool is the current standard of practice for enteric parasite diagnostics, ${ }^{12-14}$ it has been shown to be insensitive and subject to misclassification error relative to molecular methods for both protozoa and helminths. ${ }^{15-18}$ As evidence supports the adoption of molecular pathogen detection in lieu of microscopy, bulk stool remains the recommended specimen type for G. duodenalis diagnosis. ${ }^{19,20}$ For some enteropathogens, however, evidence suggests that rectal swabs may provide comparable diagnostic sensitivity with multiple potential benefits. Rectal swabs may offer greater ease of storage and transport in the absence of flammable or toxic preservatives commonly used for stool, ${ }^{21}$

*Address correspondence to Kristen Aiemjoy, Division of Infectious Diseases and Geographic Medicine, Stanford University School of Medicine, 300 Pasteur Dr., Lane Bldg., Stanford, CA 94305. E-mail: kaiemjoy@stanford.edu reduced hazardous and biohazardous waste generation during sample collection and processing, and rapid collection. ${ }^{22}$ The latter may be important for short-stay inpatient care, pediatric diagnostics, field settings, or other contexts in which sample collection and diagnostic turnaround must be streamlined. In addition, as rectal swabs are much faster and easier to obtain from young children than bulk stool while remaining minimally invasive, their use could facilitate greater child enrollment in epidemiological studies targeting this at-risk demographic.

The utility of rectal swabs for enteropathogen detection has been explored in the context of bacterial, viral, and eukaryotic disease agents. Statistically insignificant differences in the sensitivity of stool- versus swab-based PCR diagnostics have been observed for Cryptosporidium, ${ }^{23,25}$ Norovirus, ${ }^{26,27}$ Rotavirus, ${ }^{28}$ Clostridium difficile, ${ }^{29}$ and commensal bacteria of the gut microbiota. ${ }^{30,31}$ Although PCR sensitivity by rectal swabs may exceed that of bulk stool for Shigella and Campylobacter, swabs have been seen to underperform in the PCR detection of $G$. duodenalis. ${ }^{23,24}$

To further evaluate the validity of rectal swabs for the molecular detection of $G$. duodenalis, we subjected 136 paired rectal swab and bulk stool specimens from children living in southwest Niger to DNA isolation and real-time PCR (qPCR). Unlike other studies comparing the validity of rectal swab and bulk stool sample collection in clinical populations, this study focused on a populationbased representative sample and examined reproducibility by collecting duplicate samples from 50 participants.

\section{METHODS}

Study population. This study was nested within a clusterrandomized trial evaluating the effects of azithromycin mass drug administration on child mortality in rural Niger (Macrolides Oraux pour Réduire les Décès avec un Oeil sur la Résistance (MORDOR); clinicaltrials.gov NCT02048007). ${ }^{32}$ 
Study communities were located in Boboye Department, Dosso Region. Within nine of the 30 MORDOR study communities, 447 children aged $0-4$ years were randomly selected to participate in the trial, of whom 94 agreed to participate in the rectal swab sub-study.

Sample collection. On the day of the study visit, selected children and their accompanying caregivers convened in a centralized location in each community. Trained field examiners collected a rectal swab and a stool specimen from each child. The examiner first cleaned the rectal area with a disinfectant wipe and then inserted a sterile swab (FLOQSwabs ${ }^{\mathrm{TM}}$, Copan Diagnostics, Murrieta, CA) of 1-3 cm into the child's anus and gently turned the swab $180^{\circ}$. The examiner placed the swab into an empty $15 \mathrm{~mL}$ sterile tube and put the tube on ice. After swab collection, caregivers were instructed to have their children defecate in a potty chair lined with a black plastic bag. When the caregiver returned the stool to the collection station, the field examiner collected two $0.5 \mathrm{~mL}$ specimens of stool and deposited them into two empty $2 \mathrm{~mL}$ tubes. The examiner also graded the consistency of the stool sample according to the modified Bristol stool form scale for children (Supplemental Material 1). ${ }^{33}$ Among a random subset of 50 children, field examiners collected a duplicate rectal swab and/or a duplicate aliquot from the bulk stool sample, resulting in 50 duplicates of each specimen type. Both rectal swabs and bulk stool samples were immediately placed on ice and transported to $\mathrm{a}-20^{\circ} \mathrm{C}$ freezer by the end of the day. As in prior studies examining rectal swabs as a source of Giardia DNA for qPCR diagnosis, rectal swabs were preserved without media, ${ }^{22,23}$ as was bulk stool.

DNA isolation from paired bulk stool and rectal swab specimens. DNA isolation from bulk stool. Isolation of total DNA from bulk stool followed a widely used standard operating procedure optimized for the qPCR detection of enteric parasites, modified from the MP Biomedicals FastDNA ${ }^{\mathrm{TM}}$ Spin Kit for soil extraction protocol (Supplemental Material 2). All DNA isolations used the MP Biomedicals FastDNA Spin Kit and the MP Biomedicals FastPrep-24 ${ }^{\mathrm{TM}} 5 \mathrm{G}$ homogenizer (Santa Ana, CA). Each DNA isolation was spiked with $1 \mu \mathrm{L}$ of pDMD801 plasmid at a stock concentration of $100 \mathrm{pg} / \mu \mathrm{L}$, before the addition of a DNA-binding matrix solution containing silica and ceramic. This plasmid, synthesized at Smith College, served as an internal amplification control (IAC) following previously described recommendations. ${ }^{34}$ After isolation, DNA samples were stored at $-20^{\circ} \mathrm{C}$.

DNA isolation from rectal swabs. Isolation of total DNA from rectal swabs followed the protocol outlined for bulk stool, with the exception of a front-end amendment describing swab preparation and incubation preceding cell lysis (Supplemental Material 2). To develop this amendment, a literature review was performed focusing on existing rectal swab DNA isolation protocols for the detection of enteropathogens.

Before physical and chemical cell lyses, rectal swabs were removed from the $15 \mathrm{~mL}$ conical tubes used for transport and storage by grasping the swab stem with clean forceps. The swab stem was trimmed above the swab tip using clean scissors, simultaneously transferring each tip to a $2 \mathrm{~mL}$ tube. Subsequently, $1 \mathrm{~mL}$ of sodium phosphate buffer was added to each $2 \mathrm{~mL}$ tube to serve as an elution buffer for biological material on the swab surface. Based on previously described recommendations, each $2 \mathrm{~mL}$ tube was then subjected to an 18-hour incubation of gentle and continuous vortexing at room temperature. ${ }^{35}$ The remainder of the DNA isolation protocol described for bulk stool was then performed on the $1 \mathrm{~mL}$ of sodium phosphate buffer used for sample elution, and swab tips were discarded. After isolation, DNA samples were stored at $-20^{\circ} \mathrm{C}$.

Multi-parallel real-time PCR. DNA isolations were subjected to multi-parallel qPCR for the detection of $G$. duodenalis and for IAC analysis. A previously validated assay targeting the small subunit ribosomal RNA gene was used for the detection of G. duodenalis. ${ }^{36}$ Stool-derived and swab-derived DNA extracts were run on separate 96-well plates, with each sample in duplicate $7 \mu \mathrm{L}$ reactions. This study used TaqPath ProAmp Master Mix (ThermoFisher Scientific, Waltham, MA) and the Applied Biosystems ${ }^{\mathrm{TM}}$ StepOnePlus ${ }^{\mathrm{TM}}$ Real-Time PCR System (ThermoFisher Scientific, Waltham, MA). Primer oligonucleotides were synthesized by Integrated DNA Technologies, Inc. (Coralville, IA). Fluorescently labeled probes featured a 5' FAM (fluorescein) fluorophore, an internal ZEN ${ }^{T M}$ quencher, and a $3^{\prime}$ IIABkFQ quencher (Integrated DNA Technologies, Inc., Coralville, IA). Cycling conditions entailed an initial 2 -minute incubation at $50^{\circ} \mathrm{C}$, followed by a 10 -minute incubation at $95^{\circ} \mathrm{C}$ and 40 cycles of 1) a 15-second denaturation step at $95^{\circ} \mathrm{C}$ and 2) a 1-minute annealing and extension step at $60^{\circ} \mathrm{C}$ for the $\mathrm{G}$. duodenalis assay and at $59^{\circ} \mathrm{C}$ for the IAC assay.

PCR data interpretation and quality control. Samples registering exponential amplification curves with quantitation cycle $\left(\mathrm{C}_{\mathrm{q}}\right)$ values less than or equal to 38 in both duplicate wells were evaluated as positive for the respective target. ${ }^{15}$ Samples for which neither independent reaction showed amplification were deemed negative, and samples with discordant results (i.e., one independent reaction amplified, whereas the other did not) were subjected to retesting. In addition, for the IAC assay only, $C_{q}$ values for experimental samples were averaged across the sample set. All samples with duplicate mean $\mathrm{C}_{\mathrm{q}}$ values greater than two SDs above the sample set mean were assessed as suboptimal in DNA isolation quality. Any samples deemed suboptimal by the IAC assay were eliminated from the dataset.

For the G. duodenalis assay, a dilution series of genomic DNA was run as a positive control on each qPCR plate in concentrations of $10 \mathrm{pg} / \mu \mathrm{L}, 1 \mathrm{pg} / \mu \mathrm{L}$, and $100 \mathrm{fg} / \mu \mathrm{L}$. Each IAC assay plate featured a dilution series of the pDMD801 IAC plasmid in concentrations of $10 \mathrm{pg} / \mu \mathrm{L}, 100 \mathrm{fg} / \mu \mathrm{L}$, and $1 \mathrm{fg} / \mu \mathrm{L}$. Full-plate retesting was required of any qPCR plate containing positive control duplicates with a mean $\mathrm{C}_{q}$ value greater than two $\mathrm{SD}$ above the mean for the respective plasmid concentration.

No-template controls were run in quadruplicate $7 \mu \mathrm{L}$ reactions on every plate for both assays. Negative controls used $2 \mu \mathrm{L}$ of molecular biology grade water in place of $2 \mu \mathrm{L}$ DNA template, and amplification in any of the four wells elicited fullplate retesting.

Technicians performing DNA isolations and qPCR data management were masked to all metadata associated with individual samples, including identifiers linking corresponding rectal swab and stool samples or specimen pair duplicates. During DNA isolation, salient rectal swab characteristics were noted by the technician in a standardized form, including the presence of condensation in the swab storage tube and visible feces on the swab surface.

Statistical methods. We used an unweighted kappa to assess the reliability of molecular detection of $G$. duodenalis 
across duplicate bulk stool samples and duplicate rectal swabs. Following the precedent of previous studies comparing the PCR sensitivities of multiple specimen types or sametarget assays, we considered any individual who tested qPCR positive in at least one specimen replicate by bulk stool or by rectal swab to be a true positive. ${ }^{23,25,37,38}$ These true positives were used as the reference standard to calculate the sensitivity of each collection method. As the collection of human feces by two different methods would have no bearing on the species specificity of a previously validated qPCR assay, this study did not consider effects on diagnostic specificity. Given standard recommendations to collect multiple same-patient samples for maximized accuracy when diagnosing G. duodenalis, ${ }^{20}$ aggregate sensitivity when considering both duplicate specimens was also assessed.

To evaluate whether the participant's age, visible presence of stool on the swab, visible condensation in the swab storage tube, or matched stool consistency affected validity, we included these variables as covariates in multivariate logit models with the dichotomous result of the index test as the dependent variable conditional on the reference standard being positive (i.e., sensitivity).

We evaluated the relationship between rectal swab and bulk stool $C_{q}$ values in a linear regression and compared $C_{q}$ values across paired stool samples, paired rectal swabs, and the first rectal swab with the first stool sample using paired $t$-tests. All analyses were run in R v3.5.2. All code is available on GitHub (San Francisco, CA) (https://github.com/jmaasch).

Ethics statement. Ethical committees from the Niger Ministry of Health and the University of California (San Francisco, CA) granted approval for this study. We obtained verbal informed consent in French from all caregivers.

\section{RESULTS}

Population characteristics. Ninety four children participated in the study, with 86 providing paired bulk stool and rectal swab samples. Fifty provided duplicate stool samples, and 50 provided duplicate rectal swabs. The median age was 2 years (interquartile range $1-4$ ), and $58.5 \%$ (55/86) of children were female.

DNA isolation and real-time PCR quality control. All qPCR plates met the predefined quality control standards (i.e., $\mathrm{C}_{\mathrm{q}}$ values of plasmid and gDNA-positive controls were within the defined acceptable range). All DNA isolations passed IAC quality control standards with the exception of two stool samples, which were excluded from further data analysis.

Intra-method reliability. The kappa evaluating test agreement was 0.81 (95\% Cl: $0.54-1.00)$ among the 49 children with duplicate stool samples and 0.75 (95\% Cl: 0.47-1.00) among the 48 children with duplicate rectal swabs (Supplemental Table 1). Among the 31 observations with duplicate positive bulk stool samples, the mean $\mathrm{C}_{\mathrm{q}}$ value was $27.33(\mathrm{SD}=5.60)$ in replicate 1 and $27.25(S D=5.62)$ in replicate $2(P=0.647)$, with a correlation $\left(R^{2}\right)$ of 0.966 . For rectal swabs, the mean $C_{\mathrm{q}}$ value among the 22 observations with duplicate values was 27.54 $(S D=4.00)$ in replicate 1 and $26.92(S D=3.44)$ in replicate 2 $(P=0.243)$, with a correlation $\left(R^{2}\right)$ of 0.627 (Figure 1$)$.

\section{METHOD VALIDITY}

Of 84 individuals tested, 59 (70.2\%) registered positive for G. duodenalis infection in at least one specimen replicate by either sample collection method. These positives were classified as true positives for subsequent validity assessments.

Giardia duodenalis was detected in $65.5 \%$ (55/84) of bulk stool samples and $44.0 \%$ (37/84) of rectal swab samples when considering only the first replicate (Table 1). Of the 55 samples positive for G. duodenalis by bulk stool, rectal swabs failed to detect 21 (38.2\%). Three participants registered positive for G. duodenalis infection by rectal swabs and not by bulk stool. Diagnostic sensitivity for the first replicate set was $93 \%(95 \%$ Cl: $84-98)$ by bulk stool and $63 \%$ (95\% Cl: 49-75) by rectal swabs. However, if considering both specimen duplicates to simulate a multi-sample collection scenario, then bulk stool sensitivity was 95\% (95\% Cl: 86-99), whereas rectal swab sensitivity was $68 \%(95 \% \mathrm{Cl}$ : 54-79). Correlation between paired stool and swab $C_{q}$ values was low $\left(R^{2}=0.174\right)$ (Figure 1).

Binning positive bulk stool results by $C_{q}$ quartile revealed differential rectal swab performance by matched stool $\mathrm{C}_{\mathrm{q}}$ range (Figure 2). For the first quartile of bulk stool $\mathrm{C}_{q}$ values (15.95-23.51), rectal swabs were 100\% sensitive (14/14 true positives detected). Within the second and third quartiles $\left(C_{q}\right.$ 23.62-32.37), rectal swabs were $67.9 \%$ sensitive (19/28). Conversely, for bulk stool positives with $\mathrm{C}_{\mathrm{q}}$ values greater than 32.37 (i.e., samples with the lowest detectable infectious burden), rectal swab sensitivity was only $7.7 \%(1 / 13)$. When restricting to qPCR positives with bulk stool $\mathrm{C}_{\mathrm{q}}$ values less
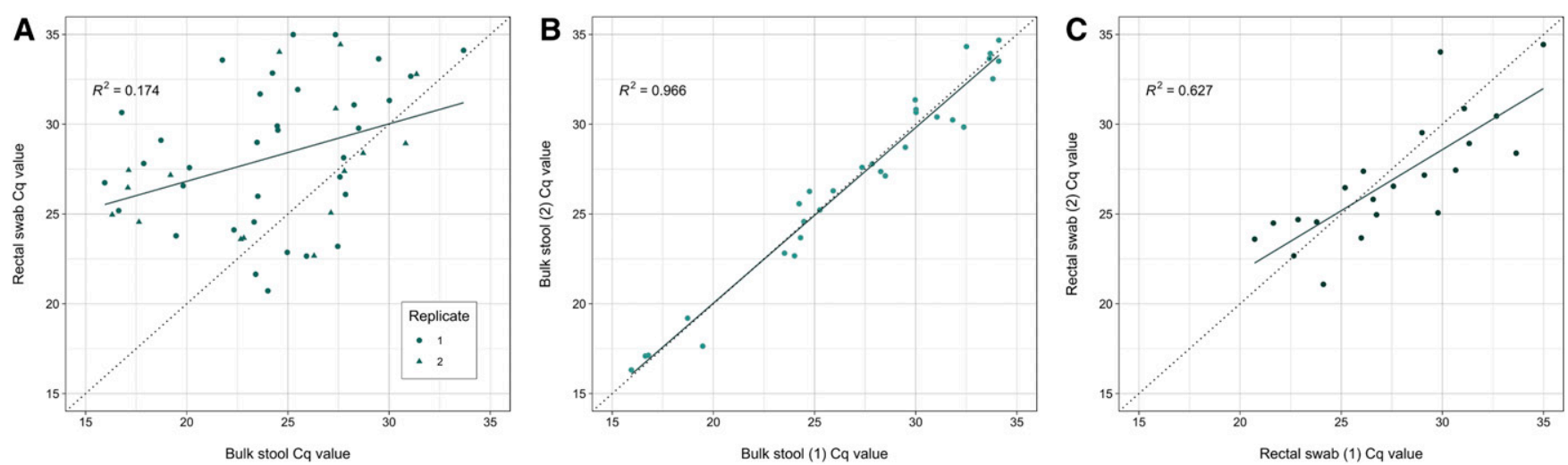

FIGURE 1. Real-time PCR quantitation cycle $\left(\mathrm{C}_{q}\right)$ value correlations by specimen type $(\mathbf{A})$ and sample replicate group $(\mathbf{B}$ and $\mathbf{C})$ for the detection of Giardia duodenalis. Plots depict $C_{q}$ value correlations for paired bulk stool and rectal swab specimens (plot $A, N=51$ ), bulk stool inter-replicate $C_{q}$ values (plot $\mathrm{B}, N=31$ ), and rectal swab inter-replicate $\mathrm{C}_{\mathrm{q}}$ values (plot $\mathrm{C}, N=22$ ). This figure appears in color at www.ajtmh.org. 
TABLE 1

Real-time PCR sensitivity by sample collection method for Giardia duodenalis detection

\begin{tabular}{lccccc}
\hline \multicolumn{1}{c}{ Specimen group } & True positive & False positive & True negative & False negative & Sensitivity, \% (95\% Cl) \\
\hline Either stool sample & 56 & 0 & 25 & 3 & $95(86-99)$ \\
Stool replicate 1 & 55 & 0 & 25 & 4 & $93(84-98)$ \\
Stool replicate 2 & 32 & 0 & 14 & 19 & $91(77-98)$ \\
Either swab sample & 40 & 0 & 25 & 22 & $68(54-79)$ \\
Swab replicate 1 & 37 & 0 & 9 & 14 & $63(49-75)$ \\
Swab replicate 2 & 25 & 0 & 9 & $64(47-79)$ \\
\hline
\end{tabular}

than or equal to 32.37 (the lowest three quartiles of stool $\mathrm{C}_{\mathrm{q}}$ ), rectal swab sensitivity in the first replicate group increased significantly to $78.0 \%$ (95\% Cl: $64-89, P<0.0001)$.

Within the first replicate, the mean $\mathrm{C}_{\mathrm{q}}$ value of bulk stool samples was $31.79(\mathrm{SD}=3.17)$ in the 21 samples with negative paired rectal swabs, compared with $24.26(S D=4.33)$ in the 34 samples with positive paired rectal swabs $(P<0.0001)$ (Figure 3). This notable mean $\mathrm{C}_{\mathrm{q}}$ difference of 7.53 cycles is equivalent to an approximate 184.8-fold difference in target DNA concentration detected by $q \mathrm{PCR}$, as a $\mathrm{C}_{\mathrm{q}}$ value increase of 1 corresponds to a doubling of PCR target (assuming 100\% efficiency). This indicates that target copy number within the original sample was, on average, 184.8 times greater for bulk stool positives with positive paired rectal swabs than for those with negative paired swabs.

Effect of covariates on rectal swab validity. Controlling for the consistency of the matched bulk stool sample, child's age, visible condensation in the swab storage tube, or the presence of visible feces on the rectal swab surface did not significantly affect diagnostic sensitivity (Supplemental Table 2). Mean $\mathrm{C}_{\mathrm{q}}$ values were slightly lower for rectal swabs with visible stool (27.5) than rectal swabs without visible stool (28.5); however, the difference was not statistically significant $(P=0.54)$.

\section{DISCUSSION}

Validity and reproducibiity. Rectal swabs detected significantly fewer true positives than did bulk stool. Sensitivity was similar to the $62.5 \%$ sensitivity observed in a prior study on the qPCR detection of $G$. duodenalis using rectal swab samples. ${ }^{23}$ Sensitivity gains from duplicate specimen testing were greater for rectal swabs than bulk stool. Although intratest agreement is higher for bulk stool than rectal swabs, these Cls overlap, and rectal swab reliability was fair.

Low fecal mass may explain inferior detection by rectal swabs, as this diminishes the likelihood of a high cyst or trophozoite count in the diagnostic sample. Additionally, the morphology, shedding patterns, or intestinal niche of G. duodenalis may not lend themselves to rectal swab detection as well as for protozoan parasites, such as, Cryptosporidium, for which rectal swabs have been reported to perform comparably to bulk stool. ${ }^{23,25}$ The intermittent shedding of $G$. duodenalis is the basis for recommendations to collect fecal samples serially and ideally over three separate days, ${ }^{20,39-41}$ as well as for the parasite's reputation as challenging to diagnose. ${ }^{19}$ In the present study, rectal swab sensitivity did improve through duplicate same-day sample collection. The molecular diagnostic

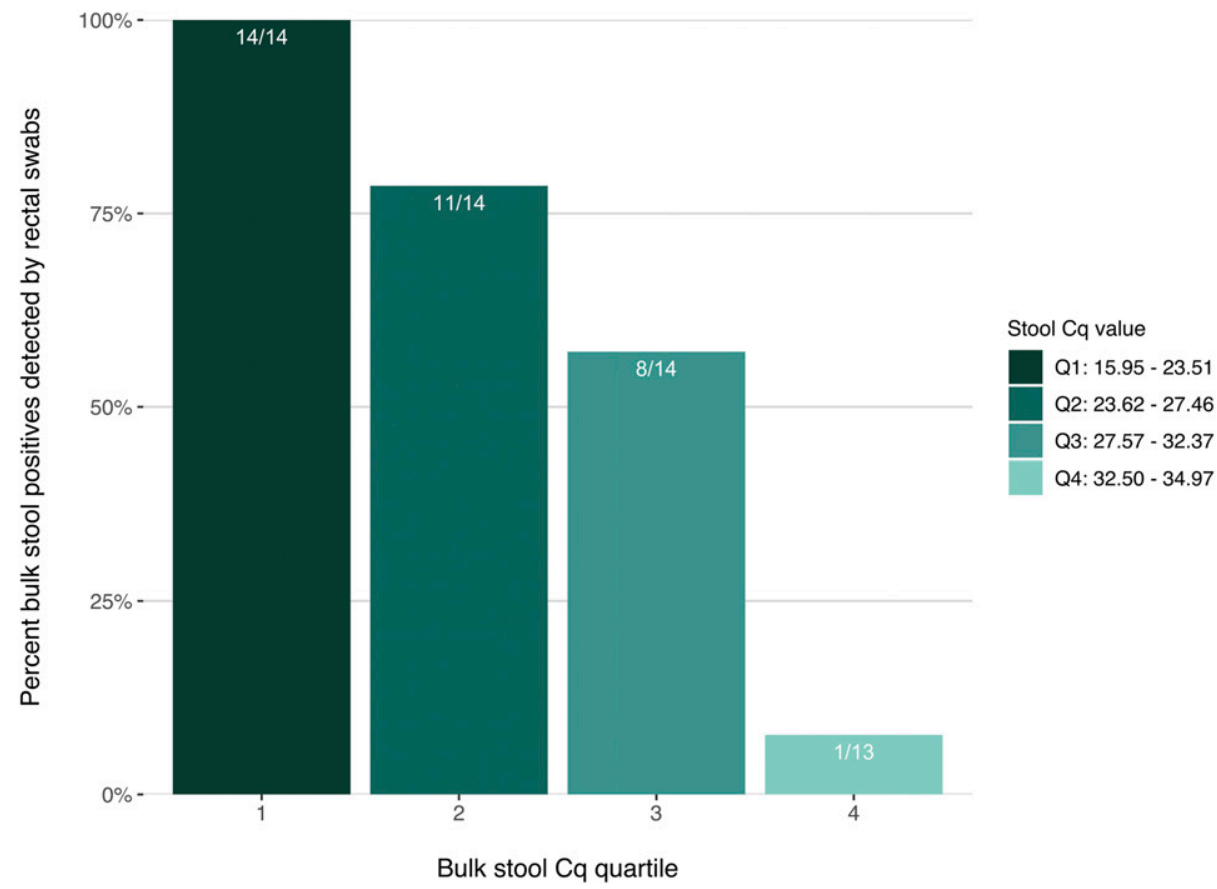

FIGURE 2. Real-time PCR sensitivity of rectal swab specimens by corresponding bulk stool quantitation cycle $\left(\mathrm{C}_{\mathrm{q}}\right)$ value. Rectal swab sensitivity varies when samples are binned by the $\mathrm{C}_{q}$ quartile of the participant's corresponding Giardia-positive bulk stool sample $(N=55)$. Data represent the first replicate group of both specimen types. This figure appears in color at www.ajtmh.org. 


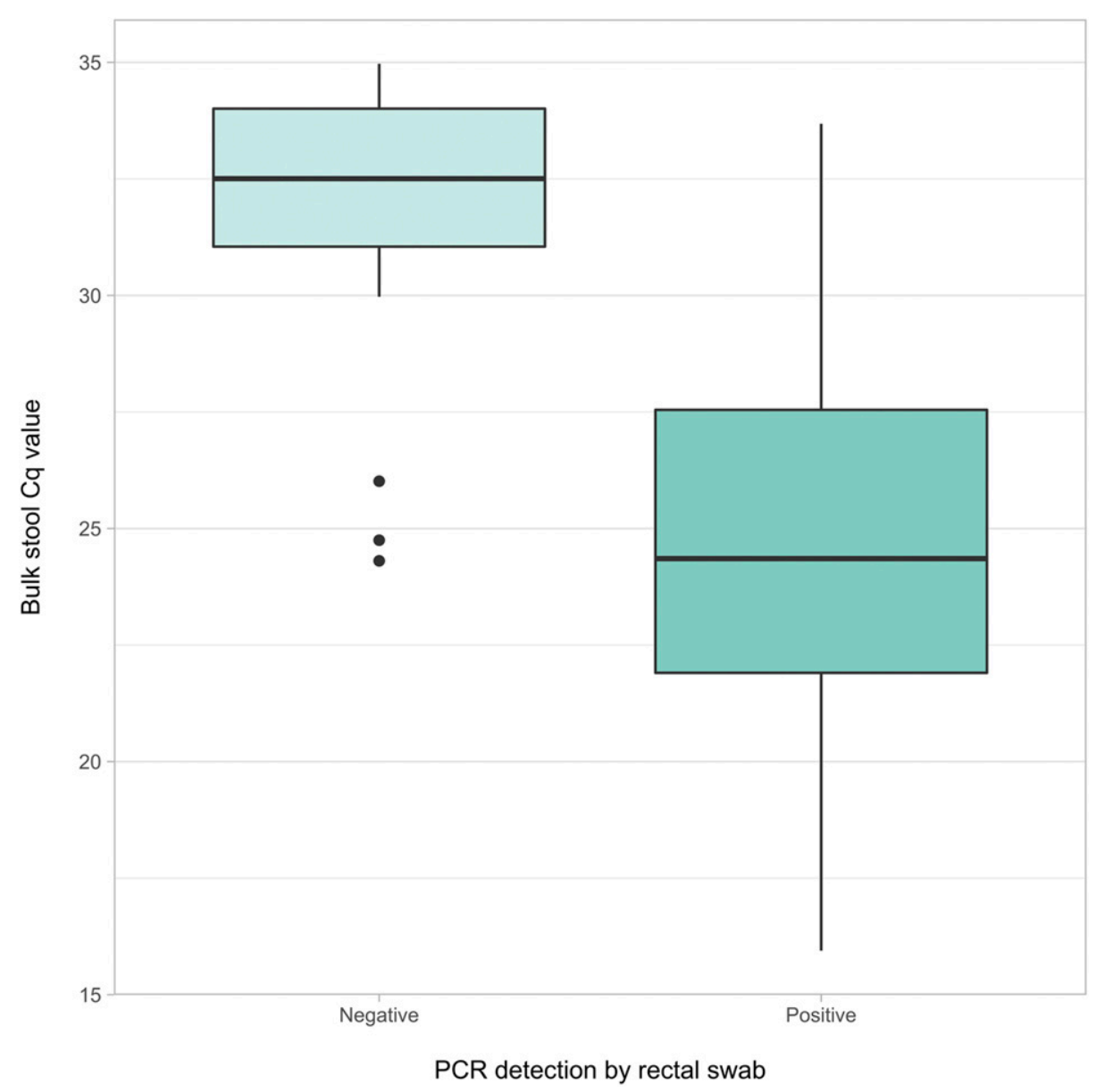

FIGURE 3. Median quantitation cycle $\left(\mathrm{C}_{\mathrm{q}}\right)$ values in bulk stool samples with corresponding qPCR-positive and qPCR-negative rectal swabs. Boxplot depicts median, upper quartile, and lower quartile $C_{q}$ values of bulk stool samples with matched rectal swabs that were positive for Giardia duodenalis $(N=34)$ and negative $(N=21)$. Data represent the first replicate group for both specimen types. Outliers were observed among qPCRnegative rectal swabs only (represented by points). This figure appears in color at www.ajtmh.org.

validity of rectal swabs collected on multiple separate days could be an area of further investigation. Furthermore, bead beating has been observed to significantly increase DNA yield from $G$. duodenalis in stool, albeit in a small sample size. ${ }^{42}$ Although the DNA isolation protocol used in the present study did include bead beating, it is possible that there are differential impacts of bead beating on swab samples versus bulk stool.

Although bulk stool is often considered a gold standard specimen type in molecular diagnostics, four individuals deemed positive for $G$. duodenalis infection by rectal swabs were deemed negative in the first bulk stool replicate, with three different positive samples missed in the second stool replicate. Although the present study demonstrates superior detection by bulk stool, it may also suggest that diagnosis by stool is not infallible. This finding may highlight the significance of temporal shedding patterns, heterogeneity of organism dispersal in a single stool specimen, rigorous laboratory and field practices (e.g., stool sample homogenization before aliquoting), and/or multiple sample collection for accurate molecular diagnosis of G. duodenalis.

Rectal swab performance may have been impacted by additional aspects of the study design, including the absence of storage media. Although this is not unprecedented among similar studies on G. duodenalis diagnosis, ${ }^{22,23}$ the performance of swabs stored with versus without preservative could be interrogated. In a study targeting $C$. difficile, qPCR detection by dry flocked swabs versus bulk stool was $100 \%$ concordant, whereas flocked swabs stored in liquid transport medium had significantly lower sensitivity. ${ }^{43}$ However, the referenced study simulated swab collection by dipping swabs in bulk stool samples.

Effect of covariates on rectal swab validity. Clinical diagnostic guidelines generally assume a positive relationship between visible soiling with fecal matter and the diagnostic utility of rectal swabs. ${ }^{44}$ However, recent interrogations of this relationship have concluded that testing rectal swabs that lack visible feces is worthwhile for the PCR detection of common enteric bacteria and viruses. ${ }^{44,45}$ Our results further corroborate this conclusion in the context of a protozoan diagnostic, as we observed no difference in rectal swab sensitivity when controlling for visible feces. There was an indication that $\mathrm{C}_{\mathrm{q}}$ values were slightly lower in rectal swabs with visible stool, although the difference was not statistically significant. However, we were likely underpowered to evaluate this effect with 24 of 86 rectal swabs having visible stool.

Whereas condensation was hypothesized to encourage enzymatic activity in media-less storage tubes, and thus to negatively affect diagnostic sensitivity, we found that visible 
moisture within media-less storage tubes did not significantly affect the qPCR detection of $G$. duodenalis by rectal swabs. However, with just 12 of 84 samples featuring visible condensation, we were underpowered to evaluate the effect of condensation on diagnostic sensitivity.

Rectal swab sensitivity by parasite load. The $\mathrm{C}_{\mathrm{q}}$ value of DNA derived from bulk stool may be considered a proxy for relative target DNA concentration and, thus, for relative pathogen load. By extension, if $\mathrm{C}_{\mathrm{q}}$ values for matched swab and stool specimens correlate strongly, then qPCR results for rectal swab samples may also be used to describe relative pathogen loads. Furthermore, if rectal swabs perform differently depending on bulk stool $\mathrm{C}_{\mathrm{q}}$ value, then this may speak to the utility of rectal swabs under different infection intensities.

Mean $\mathrm{C}_{\mathrm{q}}$ values for bulk stool and rectal swabs differed significantly, and a small proportion of variation in the rectal swab $C_{q}$ value could be explained by the $C_{q}$ value of the paired bulk stool sample, similar to a prior study. ${ }^{24}$ In addition, the $\mathrm{C}_{\mathrm{q}}$ values of rectal swab duplicates showed a less strong relationship than did stool duplicates. These findings suggest that it may be more challenging to draw conclusions about the relative pathogen load of $G$. duodenalis when performing qPCR on swab-derived DNA versus stool-derived DNA.

The sensitivity of rectal swabs increased substantially when restricting by paired stool samples with low $C_{q}$ values, an indicator of relatively high parasite burden. This phenomenon has previously been observed for $G$. duodenalis, along with several viral and bacterial targets. ${ }^{24}$ The weak performance of rectal swabs in lower pathogen load scenarios may be problematic in population-based studies and monitoring programs, where lower level infections may go undetected while still contributing to morbidity, parasite transmission, and recrudescence. ${ }^{46-49}$ Conversely, this may be an advantage for studies interested in evaluating the morbidity effects of higher burden infections on growth, cognitive stunting, or other significant indicators in childhood development. Using rectal swabs to detect higher burden infections may also be useful for studies assessing intervention effects targeted at higher burden individuals.

Whether the high- $\mathrm{C}_{\mathrm{q}}$ true positives missed by rectal swabs represent subclinical infections or nonpathogenic colonization could be an area of further investigation. It has been suggested that this may be the case, as organisms which adhere to mucosae and thus may be pathogenic are more likely to be detected by swabs than are organisms within the intestinal lumen, which may be abundant in stool. ${ }^{22}$

\section{CONCLUSION}

Molecular diagnostics for enteric parasites like G. duodenalis are increasingly common and typically require bulk stool specimens. Relative to bulk stool, rectal swabs may offer greater ease of collection, storage, transport, and waste disposal. However, the significant loss of diagnostic sensitivity as documented in this study could lead to underestimation of G. duodenalis prevalence when using rectal swabs. Given the differential performance of swabs for higher versus lower level infections, this would be problematic in contexts where lowburden infections are common. Poor detection of lower level infections may also be detrimental in control and elimination scenarios, where undetected low-burden infections may still contribute to parasite transmission and recrudescence. Conversely, the fair sensitivity of rectal swabs for diagnosing higher level infections may indicate the utility of swabs as a frontline sampling method in contexts where high-burden infections are most relevant, particularly when bulk stool collection is logistically or financially infeasible.

Received December 6, 2019. Accepted for publication May 2, 2020.

Published online June 8, 2020.

Note: Supplemental materials, figure, and tables appear at www. ajtmh.org.

Acknowledgments: We would like to thank the children and guardians who participated in this study for their time and invaluable contributions. We would like to thank Rojelio Mejia (Baylor College of Medicine, Houston, TX) for his work developing the fecal DNA isolation protocol from which the rectal swab DNA isolation protocol was adapted.

Financial support: This study was supported by the Bill \& Melinda Gates Foundation (OPP1032340) and the University of California San Francisco (UCSF) Catalyst Program.

Disclosure: The Salesforce Foundation provided user licenses to Salesforce.com and cloud storage.

Authors' addresses: Jacqueline R. M. A. Maasch, Department of Computer and Information Science, University of Pennsylvania School of Engineering and Applied Science, Philadelphia, PA, E-mail: jmaasch@seas.upenn.edu. Ahmed M. Arzika, The Carter Center, Niamey, République du Niger, E-mail: mamaneahmed@yahoo.fr. Catherine Cook, Elodie Lebas, Jeremy D. Keenan, and Thomas M. Lietman, Proctor Foundation, University of California San Francisco, San Francisco, CA, E-mails: catherine.cook@ucsf.edu, elodie.lebas@ ucsf.edu, jeremy.keenan@ucsf.edu, and tom.lietman@ucsf.edu. Nils Pilotte and Steven A. Williams, Department of Biological Sciences, Smith College, Northampton, MA, E-mails: npilotte@smith.edu, jgrant@smith.edu, and swilliam@smith.edu. Kristen Aiemjoy, Division of Infectious Diseases and Geographic Medicine, Department of Medicine, Stanford University School of Medicine, CA, E-mails: kaiemjoy@stanford.edu and kaiemjoy@stanford.edu.

This is an open-access article distributed under the terms of the Creative Commons Attribution (CC-BY) License, which permits unrestricted use, distribution, and reproduction in any medium, provided the original author and source are credited.

\section{REFERENCES}

1. Ankarklev J, Jerlstrom-Hultqvist J, Ringqvist E, Troell K, Svard SG, 2010. Behind the smile: cell biology and disease mechanisms of Giardia species. Nat Rev Microbiol 8: 413-422.

2. Faghiri Z, Widmer G, 2011. A comparison of the Giardia lamblia trophozoite and cyst transcriptome using microarrays. BMC Microbiol 11: 91

3. Einarsson E, Ma'ayeh S, Svärd SG, 2016. An up-date on Giardia and giardiasis. Curr Opin Microbiol 34: 47-52.

4. Heyworth MF, 2016. Giardia duodenalis genetic assemblages and hosts. Parasite 23: 13.

5. Cacciò SM, Lalle M, Svärd SG, 2018. Host specificity in the Giardia duodenalis species complex. Infect Genet Evol 66: 335-345.

6. Centers for Disease Control and Prevention, 2015. Giardia. Available at: https://www.cdc.gov/parasites/giardia/index.html. Accessed June 23, 2019.

7. Oyerinde JPO, Ogunbi O, Alonge AA, 1977. Age and sex distribution of infections with Entamoeba histolytica and Giardia intestinalis in the lagos population. Int J Epidemiol 6: 231-234.

8. Greig JD, Michel P, Wilson JB, Lammerding AM, Majowicz SE, Stratton J, Aramini JJ, Meyers RK, Middleton D, McEwen SA, 2001. A descriptive analysis of giardiasis cases reported in Ontario, 1990-1998. Can J Public Health 92: 361-365.

9. Snel SJ, Baker MG, Kamalesh V, French N, Learmonth J, 2009. A tale of two parasites: the comparative epidemiology of cryptosporidiosis and giardiasis. Epidemiol Infect 137: 1641-1650.

10. Painter JE, Gargano JW, Collier SA, Yoder JS, 2015. Giardiasis surveillance - United States, 2011-2012. MMWR Morb Mortal Wkly Rep 64: 15-25. 
11. Seguí $R$ et al., 2018. Prevalence of intestinal parasites, with emphasis on the molecular epidemiology of Giardia duodenalis and Blastocystis sp., in the Paranaguá Bay, Brazil: a community survey. Parasite Vector 11: 490.

12. Koehler AV, Jex AR, Haydon SR, Stevens MA, Gasser RB, 2014. Giardia/giardiasis-a perspective on diagnostic and analytical tools. Biotechnol Adv 32: 280-289.

13. Nikolay B, Brooker SJ, Pullan RL, 2014. Sensitivity of diagnostic tests for human soil-transmitted helminth infections: a metaanalysis in the absence of a true gold standard. Int $J$ Parasitol 4: 765-774.

14. World Health Organization, 2002. Prevention and control of schistosomiasis and soil-transmitted helminthiasis. World Health Organ Tech Rep Ser 912: i-vi, 1-57.

15. Mejia R, Vicuña $Y$, Broncano N, Sandoval C, Vaca M, Chico M, Cooper PJ, Nutman TB, 2013. A novel, multi-parallel, real-time polymerase chain reaction approach for eight gastrointestinal parasites provides improved diagnostic capabilities to resource-limited at-risk populations. Am J Trop Med Hyg 88: 1041-1047.

16. Easton AV et al., 2016. Multi-parallel qPCR provides increased sensitivity and diagnostic breadth for gastrointestinal parasites of humans: field-based inferences on the impact of mass deworming. Parasit Vectors 9: 38

17. Sánchez A et al., 2017. Molecular epidemiology of Giardia, Blastocystis and Cryptosporidium among indigenous children from the Colombian Amazon Basin. Front Microbiol 8: 248.

18. Benjamin-Chung $\mathrm{J}$ et al., 2020. Comparison of multi-parallel qPCR and Kato-Katz for detection of soil-transmitted helminth infection among children in rural Bangladesh. PLoS Negl Trop Dis 14: e0008087.

19. Johnston SP, Ballard MM, Beach MJ, Causer L, Wilkins PP, 2003. Evaluation of three commercial assays for detection of Giardia and Cryptosporidium organisms in fecal specimens. J Clin Microbiol 41: 623-626.

20. Centers for Disease Control and Prevention, 2015. Giardia: Diagnosis \& Detection. Available at: https://www.cdc.gov/ parasites/giardia/diagnosis.html. Accessed June 23, 2019.

21. Papaiakovou M, Pilotte N, Baumer B, Grant J, Asbjornsdottir K, Schaer F, Hu Y, Aroian R, Walson J, Williams SA, 2018. A comparative analysis of preservation techniques for the optimal molecular detection of hookworm DNA in a human fecal specimen. PLoS Negl Trop Dis 12: e0006130.

22. Freedman SB et al., 2017. Enteropathogen detection in children with diarrhoea, or vomiting, or both, comparing rectal flocked swabs with stool specimens: an outpatient cohort study. Lancet Gastroenterol Hepatol 2: 662-669.

23. Goldfarb DM et al., 2014. Evaluation of anatomically designed flocked rectal swabs for molecular detection of enteric pathogens in children admitted to hospital with severe gastroenteritis in Botswana. J Clin Microbiol 52: 3922-3927.

24. Liu J et al., 2016. Optimization of quantitative PCR methods for enteropathogen detection. PLoS One 11: e0158199.

25. Kabayiza J-C, Andersson ME, Welinder-Olsson C, Bergström T, Muhirwa G, Lindh M, 2013. Comparison of rectal swabs and faeces for real-time PCR detection of enteric agents in Rwandan children with gastroenteritis. BMC Infect Dis 13: 447.

26. Arvelo W, Hall AJ, Estevez A, Lopez B, Gregoricus N, Vinjé J, Gentsch JR, Parashar U, Lindblade KA, 2013. Diagnostic performance of rectal swab versus bulk stool specimens for the detection of rotavirus and norovirus: implications for outbreak investigations. J Clin Virol 58: 678-682.

27. Sidler JA, Käch R, Noppen C, Dangel M, Battegay M, Bingisser R, Dubuis O, Widmer AF, 2014 Rectal swab for detection of norovirus by real-time PCR: similar sensitivity compared to faecal specimens. Clin Microbiol Infect 20: O1017-01019.

28. Gibory M, Haltbakk I, Flem E, Vainio K, Salamanca BV, Størdal K, Nordbø SA, Jakobsen K, Haarr E, Dudman SG, 2017. Rotavirus detection in bulk stool and rectal swab specimens in children with acute gastroenteritis in Norway. J Clin Virol 97: 50-53.

29. Kundrapu S, Sunkesula VCK, Jury LA, Sethi AK, Donskey CJ, 2012. Utility of perirectal swab specimens for diagnosis of Clostridium difficile infection. Clin Infect Dis 55: 1527-1530.
30. Budding AE, Grasman ME, Eck A, Bogaards JA, VandenbrouckeGrauls CMJE, van Bodegraven AA, Savelkoul PHM, 2014. Rectal swabs for analysis of the intestinal microbiota. PLoS ONE 9: e101344.

31. Bassis CM, Lolans K, Seekatz AM, Weinstein RA, Young VB, Hayden MK, 2017. Comparison of stool versus rectal swab samples and storage conditions on bacterial community profiles. BMC Microbiol 17: 78.

32. Keenan JD et al., 2018. Azithromycin to reduce childhood mortality in sub-Saharan Africa. N Engl J Med 378: 1583-1592.

33. Chumpitazi BP, Lane MM, Czyzewski DI, Weidler EM, Swank PR, Shulman RJ, 2010. Creation and initial evaluation of a stool form scale for children. J Pediatr 157: 594-597.

34. Deer DM, Lampel KA, González-Escalona N, 2010. A versatile internal control for use as DNA in real-time PCR and as RNA in real-time reverse transcription PCR assays. Lett App/ Microbiol 50: 366-372.

35. Adamowicz MS, Stasulli DM, Sobestanovich EM, Bille TW, 2014. Evaluation of methods to improve the extraction and recovery of DNA from cotton swabs for forensic analysis. PLoS One 9: e116351.

36. Verweij JJ, Schinkel J, Laeijendecker D, van Rooyen MAA, van Lieshout L, Polderman AM, 2003. Real-time PCR for the detection of Giardia lamblia. Mol Cell Probe 17: 223-225.

37. Gustavsson L, Westin J, Andersson L-M, Lindh M, 2011. Rectal swabs can be used for diagnosis of viral gastroenteritis with a multiple real-time PCR assay. J Clin Virol 51: 279-282.

38. Pilotte N, Maasch JRMA, Easton AV, Dahlstrom E, Nutman TB, Williams SA, 2019. Targeting a highly repeated germline DNA sequence for improved real-time PCR-based detection of Ascaris infection in human stool. PLoS Negl Trop Dis 13: e0007593.

39. Hiatt RA, Markell EK, Ng E, 1995. How many stool examinations are necessary to detect pathogenic intestinal protozoa? Am J Trop Med Hyg 53: 36-39.

40. Hooshyar H, Rostamkhani P, Arbabi M, Delavari M, 2019. Giardia lamblia infection: review of current diagnostic strategies. Gastroenterol Hepatol Bed Bench 12: 3-12.

41. Uchôa FFdeM, Sudré AP, Macieira DdeB, Almosny NRP, 2017. The influence of serial fecal sampling on the diagnosis of giardiasis in humans, dogs, and cats. Rev Inst Med Trop São Paulo 59: e61.

42. Elwin K, Fairclough HV, Hadfield SJ, Chalmers RM, 2014. Giardia duodenalis typing from stools: a comparison of three approaches to extracting DNA, and validation of a probe-based real-time PCR typing assay. J Med Microbiol 63: 38-44.

43. Jazmati N, Kirpal E, Piepenbrock E, Stelzer Y, Vehreschild MJGT, Seifert H, 2018. Evaluation of the use of rectal swabs for laboratory diagnosis of Clostridium difficile infection. $J$ Clin Microbiol 56: e00426-18.

44. Glisovic S, Eintracht S, Longtin Y, Oughton M, Brukner I, 2018. Rectal swab screening assays of public health importance in molecular diagnostics: sample adequacy control. J Infect Public Health 11: 234-237.

45. Xie J et al., 2019. Pigment visibility on rectal swabs used to detect enteropathogens: a prospective cohort study. J Clin Microbiol 57: e00213-19.

46. Boots M, Greenman J, Ross D, Norman R, Hails R, Sait S, 2003. The population dynamical implications of covert infections in host-microparasite interactions. J Anim Ecol 72: 1064-1072.

47. Burden JP, Nixon CP, Hodgkinson AE, Possee RD, Sait SM, King LA, Hails RS, 2003. Covert infections as a mechanism for longterm persistence of baculoviruses. Ecol Lett 6: 524-531.

48. Chappell CL, Okhuysen PC, Sterling CR, DuPont H, 1996. Cryptosporidium parvum: intensity of infection and oocyst excretion patterns in healthy volunteers. J Infect Dis 173: 232-236.

49. McCallum H, Fenton A, Hudson PJ, Lee B, Levick B, Norman R, Perkins SE, Viney M, Wilson AJ, Lello J, 2017. Breaking beta: deconstructing the parasite transmission function. Philos Trans $R$ Soc Lond B Biol Sci 372: 20160084. 